ÉDITIONS J. REY - B. ARTHAUD, Editeur, GRENOBLE

\author{
Abonnement pour une Année $\left\{\begin{array}{l}\text { France...... } 40 \text { francs } \\ \text { Étranger... } 50 \text { francs }\end{array}\right\}$ Le Numéro : 7 francs
}

Compte Chèques Postaux LYON 5-84

SOMMAIRE

HYDRAULIQUE. - Conditions techniques d'établissement des conduites forcées, par Georges FERrand, Diresteur de la Société Dauphinoise d'Etudes et de Montages, Ingénieur aux Etablissements Bouchayer \& Viallet.- Sur une loi de l'écoulement en canal découvert, par M. Montagné, ingénieur, ancien élève de l'Institut polytechnique de Grenoble.

ÉLECTRICITÉ. - Comparaison entre le courant continu et le courant alternatif, par M. MATHivet, ingénieur en chef des travaux du jour aux Mines de Vicoigne, Noux et Drocourt.

LÉGISLATION. - Le courant électrique importé de Suisse et la taxe d'importation. Arrêt de la Cour de Cassation du 5 juillet 1927, par Paul Bougault, avocat à la Cour d'Appel de Lyon.

\title{
HYDRAULIQUE
}

\section{Conditions techniques d'établissement des conduites forcées}

par M. Georges Ferrand, Directeur de la Société Dauphinoise d'Etudes et de Montages Ingénieur aux Elablissements Bouchayer \& Viallet

\section{Installation des conduites forcées}

\section{I. - Piguetage des conduites forcées}

La détermination sur le terrain du meilleur tracé d'une conduite forcée constitue une opération délicate qui dépend en grande partie de l'expérience, du jugement et de l'esprit d'observatiou de l'ingénieur chargé de ce travail.

Il ne nous paraît pas possible de fixer d'une façon absolue les éléments nécessaires à l'établissement de ce "piquetage ". Nous indiquerons toutefois quelques considérations générales dont on devra tenir comple pour l'exécution de ce travai

La conduite à installer doil coûler naturellement le moins cher possible. Par conséquent, il faut lui domer

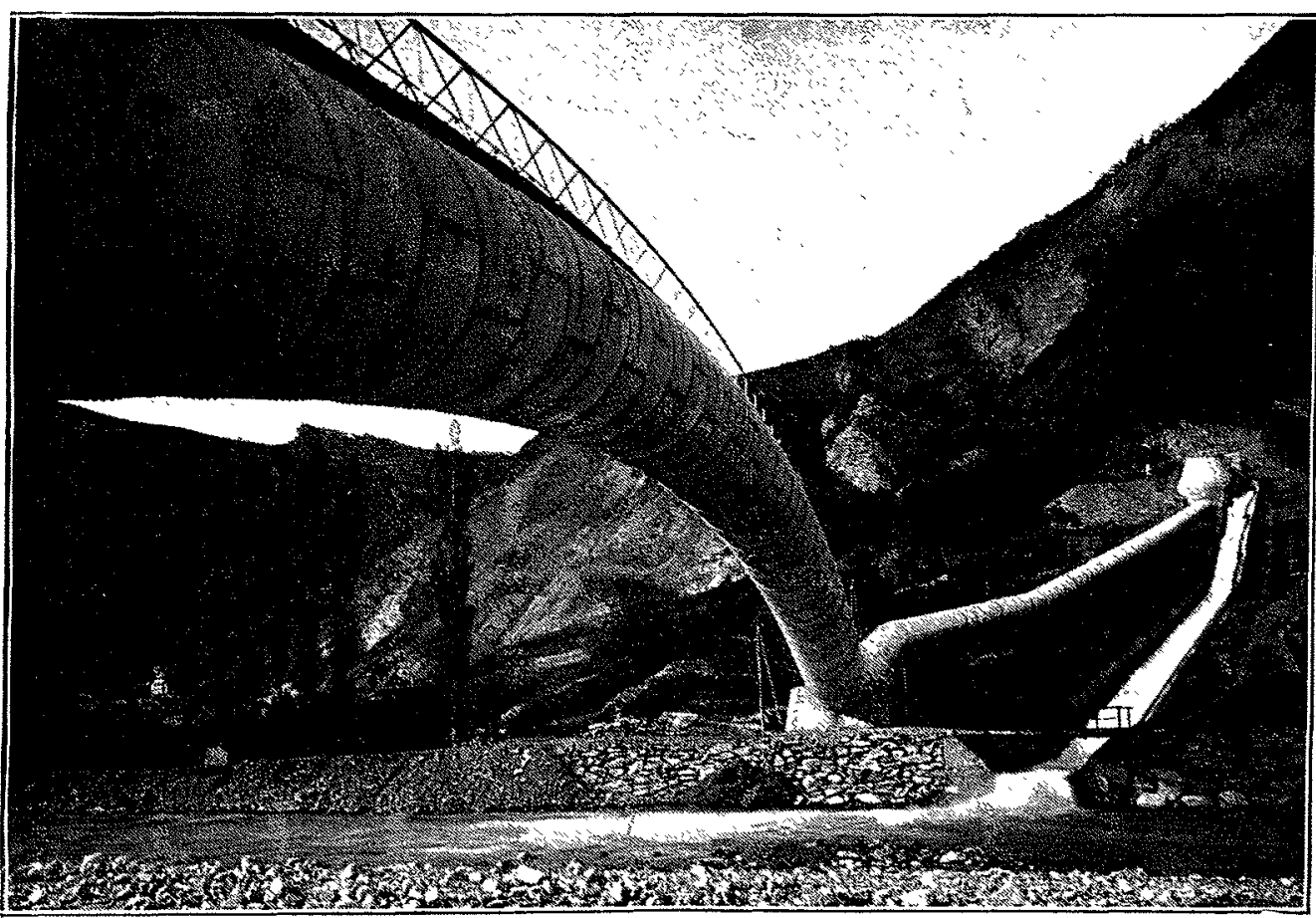

Traversée en arc à Saint-Jean-de-Maurienne (Savoie) faut donc dans le cas de profils accidentés prévoir un tracé rectiligne en plan permettant l'installation d'un câble transporteur pour amener les éléments de conduite à leur emplacement de montage. Un plan incliné peut s'installer le long d'un tracé sinueux en plan mais ce système est à éviter lorsque la pente générale des terrains traversés dépasse $50 \%$. sa longueur minimum et observer qu'elle sera d'autant moins lourde que les plus fortes pentes se trouveront dans les parties inlérieures du tracé.

L'approvisionnement des tuyaux à pied-d'œuvre et le montage doivent pouvoir s'effectuer avec le minimum de difficulté. Il forcée, les caux devront pouvoir s'écouler sans inconvénient pour l'usine génératrice.

Il faudra s'assurer aussi que la conduite reposera sur un sol stable et qu'elle sera à l'abri des éboulements, avalanches, etc...

On voit que le problème est assez complexe. Il laisse une grande 
marge à l'appréciation de l'Ingénieur chargé de son étude. Il sera en pratique, très souvent simplifié du fait que pour des raisons capitales on aura été obligé de fixer a priori les emplacements de la chambre de mise en charge et de l'usine génératrice, c'est-à-dire les points de départ et d'arrivée de la conduite.

Il serait d'ailleurs bien préférable que la position de ces points fixes ne soit arrètée qu'après entente avec l'homme de mélier.

\section{II. - Implantation des Conduites}

Doit-on enterrer les conduites ou doit-on les établir hors-sol ?

Cette question est très discutée et chaque méthode possède ses avantages et ses inconvénients.

Une conduite à air libre peut être surveillée, visitée ou entretenue facilement; elle se trouve par contre exposée aux grandes variations de température, aux éboulements, aux intempéries, il faut en hiver prendre de grandes précautions pour en éviter une conduite lout au moins en certains endroits, notamment en haute montagne. En d'autres circonstances, la conduite à air libre paraîra plus avantageuse parce qu'il serait trop cốteux de crenser des tranchees on de faire tenir des rembl is. Il s'agit donc de cas d'espèces qui devront èlre étudiés, sur place, très minulieusement en meltant en balance les avantages el les inconvénients de chaque méthode. Bien souvent d'ailleurs en employant la formule mixle, on obtiendra le meilleur résultal. C'esl une question épineuse qui a loujours divisé les hydrauliciens! Il semble bien cependanl à l'heure actuelle, que l'idée des conduites enlerrées tend à se développer et nous ajouterons que nous sommes partisans d'adopler celte méthode toutes les fois quion le pourra.

\section{III. -- ANCRAgES ET RACCOLDEMENTS DES CONDUITES}

Lorsque les conduites se trouvent placées hors sol il y a lieu de

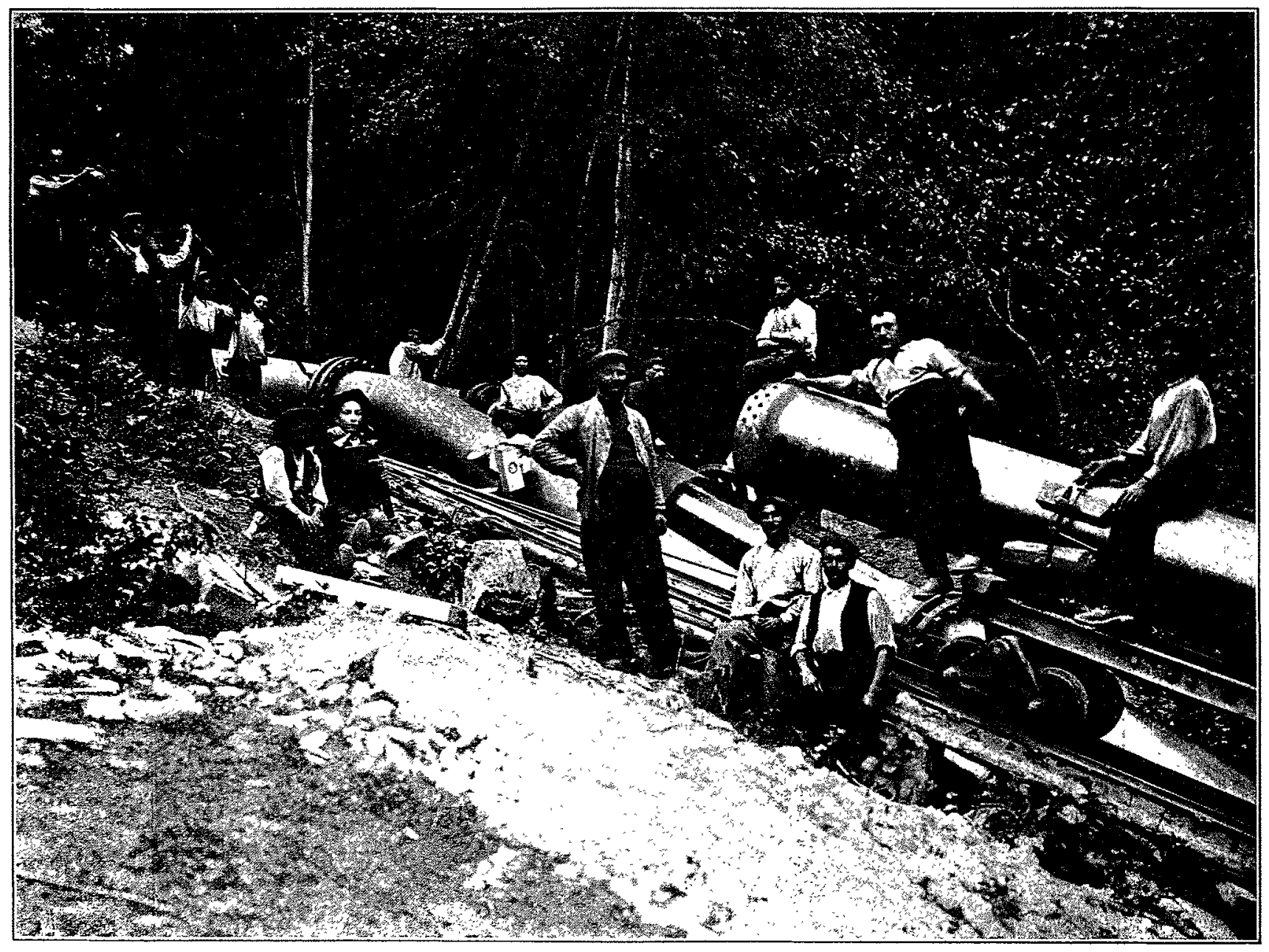

Montage par plan incliné

le gel et la protéger contre les méfaits de la neige. Les travaux de maçonnerie nécessaires pour sa stabilité sont très importants et par suite très coûteux.

Avec une conduite enterrée, on obtient à peu de frais une stabilité parfaite, elle se trouve complètement protégée contre les phénomènes extérieurs mais sa surveillance et son entretien sont à peu près impossibles. - On a cependant constaté pratiquement qu'une conduite enterrée, bien peinte ay goudron n'a pas beaucoup à craindre l'oxydation. Nous en connaissons certaines qui sont installées depuis de nombreuses années et qui n'ont donné lieu à aucun mécompte. Les systèmes de joints employés actuellement donnent également toute sécurité et leur entretien est nul.

Dans certains cas, on sera amené obligatoirement à enterrer prendre des précautions spéciales d'une importance capitale pour leur stabilité. Il faut, en effet, éviter que par suite des variations de température il se développe des efforts anormaux dans leur sens longitudinal.

Deux méthodes sont en présence :

La méthode dite "Suisse " et la méthode dite "Française ".

La méthode Suisse consiste à ancrer à l'aide de massifs en maçonneries tous les coudes sans exception et à placer entre ces massifs des "joints de dilatation " destinés à permettre entre les points fixes ainsi constitués, la libre dilatation de la conduite.

Ces appareils constilués comme indiqué sur la fig. 1 sont délicats, ils nécessitent beaucoup de surveillance et d'entretien et leur fonctionnement n'est pas toujours sûr ainsi 
que l'expérience nous l'a montré. En cas d'accident (qui se trouverait localisé dans la méthode française sur quelques mètres de tuyaux seulement) la présence de ces appareils peut donner lieu dans certains cas à des accidents intéressant de grandes longueur de conduite.

En France, on n'utilise généralement pas les joints de dilatation que l'on considère comme une source d'ennuis et dont l'installation est onéreuse.

On préfère adopter l'autre méthode consistant à réaliser le long de la conduite quelques points fixes à l'aide de massifs d'ancrage en maçonnerie. Les efforts longitudinaux provenant

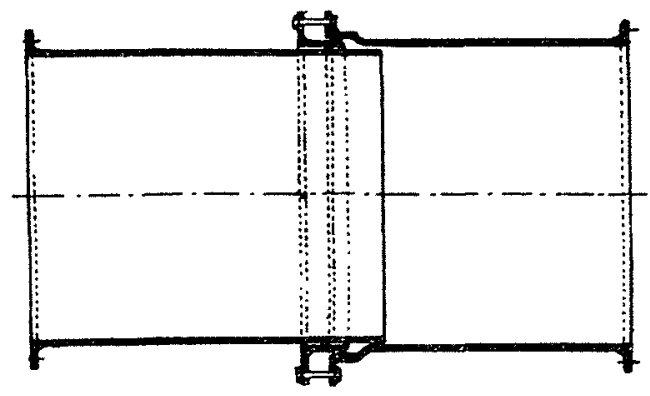

Fig. 1

des variations de température sont équilibrés par le travail moléculaire du métal. Ce travail moléculaire se trouve d'ailleurs d'autant plus diminué qu'on aura laissé libres le plus grand nombre de coudes possible entre les points fixes; ceux-ci par leur élasticité favorisent la dilatation de la conduite.

Ainsi que le dit M. Eydoux dans un de ses ouvrages (1) : "Dans ce cas l'étude de la stabilité de la conduite repose entiè" rement sur des écarts de température. Il faut en corséquence " que la conduite soit " arrêtée " à une température absolument " fixée d'avance et correspondant aux calculs."

Il y a lieu de remarquer à ce sujet que les effets de la dilatation se produisent surtout lorsque la conduite est vide. Lorsqu'elle est pleine la grande masse d'eau en mouvement fait que la paroi de la conduite ne suit pas les variations de températures extérieures et prend celle de l'eau que l'on peut considérer pratiquement comme constante.

La température de " raccordement " doit être aussi voisine que possible de celle de l'eau de telle façon que la conduite en service n'ait à supporter que les efforts résultant de la pression intérieure.

Pour arriver à ce résultat il y a lieu de prendre au moment du montage des dispositions particulières. Pour raccorder la conduite entre les points fixes, on emploie couramment : soit des viroles de raccordement rivées dans le cas de tuyaux assemblés sur place par rivure, soit des manchettes à brides dans le cas de tuyaux assemblés sur place par brides.

Les longueurs de ces viroles ou manchettes sont déterminées sur place à la fin du montage. Les mesures doivent en être prises et leur mise en place effectuée à la température convenable, ce qui n'est pas toujours facile et" nécessite une grande expérience de la part des monteurs.

Pour rendre ce travail de raccordement moins délicat on emploie souvent des appareils appelés "joints de réglage" (fig. 2) qui sont des espèces de joints de dilatation permettant à la conduite de se dilater librement pendant toute la durée du montage et que l'on immobilise au moment de la mise en service.

(1) Hydraulique industrielle et usines hydrauliques de la conduite à la température voulue à l'aide de brides et boulons convenablement disposés. Ce dispositif possède l'inconvénient de contenir un presse-étoupes que l'on est obligé de surveiller et d'entretenir fréquemment.

Pour supprimer cet ennui, nous avons étudié, il y a quelques années, un système spécial d'ancrage, dit " ancrage de réglage * (fig. 3), qui remplit exactement le même rôle qu'un joint de réglage mais dont l'inconvénient signalé est supprimé.

Il est constitué de la façon suivante :

Dans les massifs d'ancrage, la conduite peut coulisser à l'intérieur d'une chemise en tôle scellée dans la maçonnerie. Cette

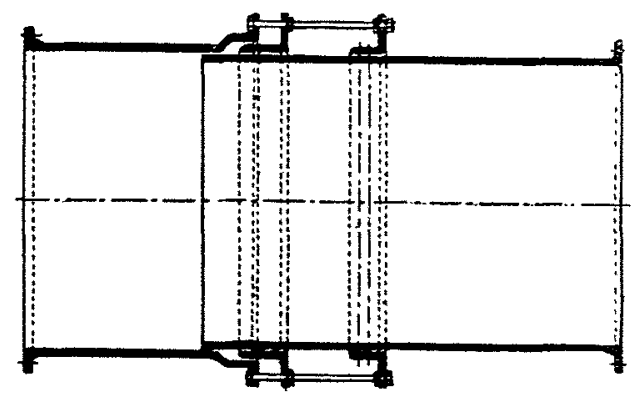

Fig. 2 - Joint de réglage

chemise et le tuyau peuvent être rendus solidaires à volonté à l'aide de deux brides, l'une fixée sur la chemise, l'autre fixée sur le tuyau, la fixation étant obtenue à l'aide de boulons réunissant les deux brides.

En cours de montage la conduite coulisse librement à l'intérieur de la chemise et la fixation est obtenue au moment de la

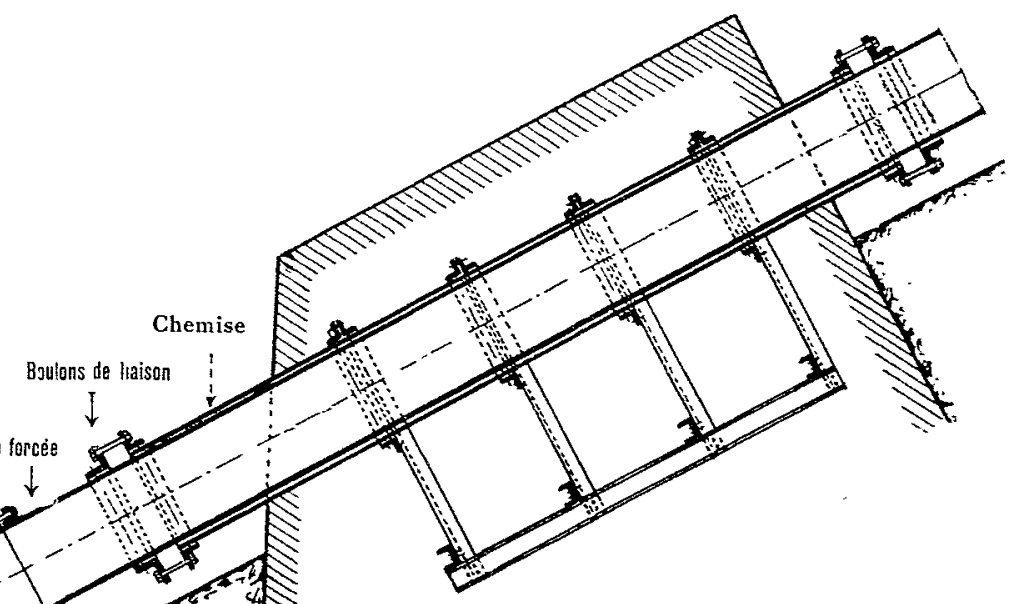

Fig. 3. - Ancrage de réglage

mise en service par la mise en place à la température convenable des boulons de liaison. Vers les points de départ de montage, la mise en place des manchettes de raccordement se trouve grandement facilitée du fait que la couronne de boulons permet le déplacement des tronçons à raccorder.

Ce système a été installé à l'usine du Rivier-d'Allemont et à l'usine d'Eget où il a donné les meilleurs résultats. La conduite elle-mếme ne possède aucune cornière d'ancrage rivée ce qui supprime totalement les chances de fuite à l'intérieur des massifs ; d'autre part, la solidarité de la conduite et des massifs n'ayant lieu qu'à la fin du montage, ceux-ci ont tout le temps de faire une bonne prise ce qui leur empêche de se fissurer en cours de montage comme cela se produit généralement.

La position des massifs d'ancrage le long de la conduite se détermine par le calcul de telle sorte qu'avec les différences 
d'épaisseurs des luyaux entre deux massils, la laligue du luyau le plus mince ne dépasse pas un laux de travail normal dans le cas le plus défavorable, c'est-à-dire lorsque la conduite est vide à la temperature la plus éloignée de la tempéralure de raccordement. En pratique, on concilie les positions théoriques avec la nécessité d'oblenir des massils d'ancrage élablis sur de bonnes fondations.

\section{IV. - Stpponts Des Condutes}

Quelle que soit la méthode emplorée, française ou suisse, la conduite est supporlée entre deux massifs d'ancrage par des

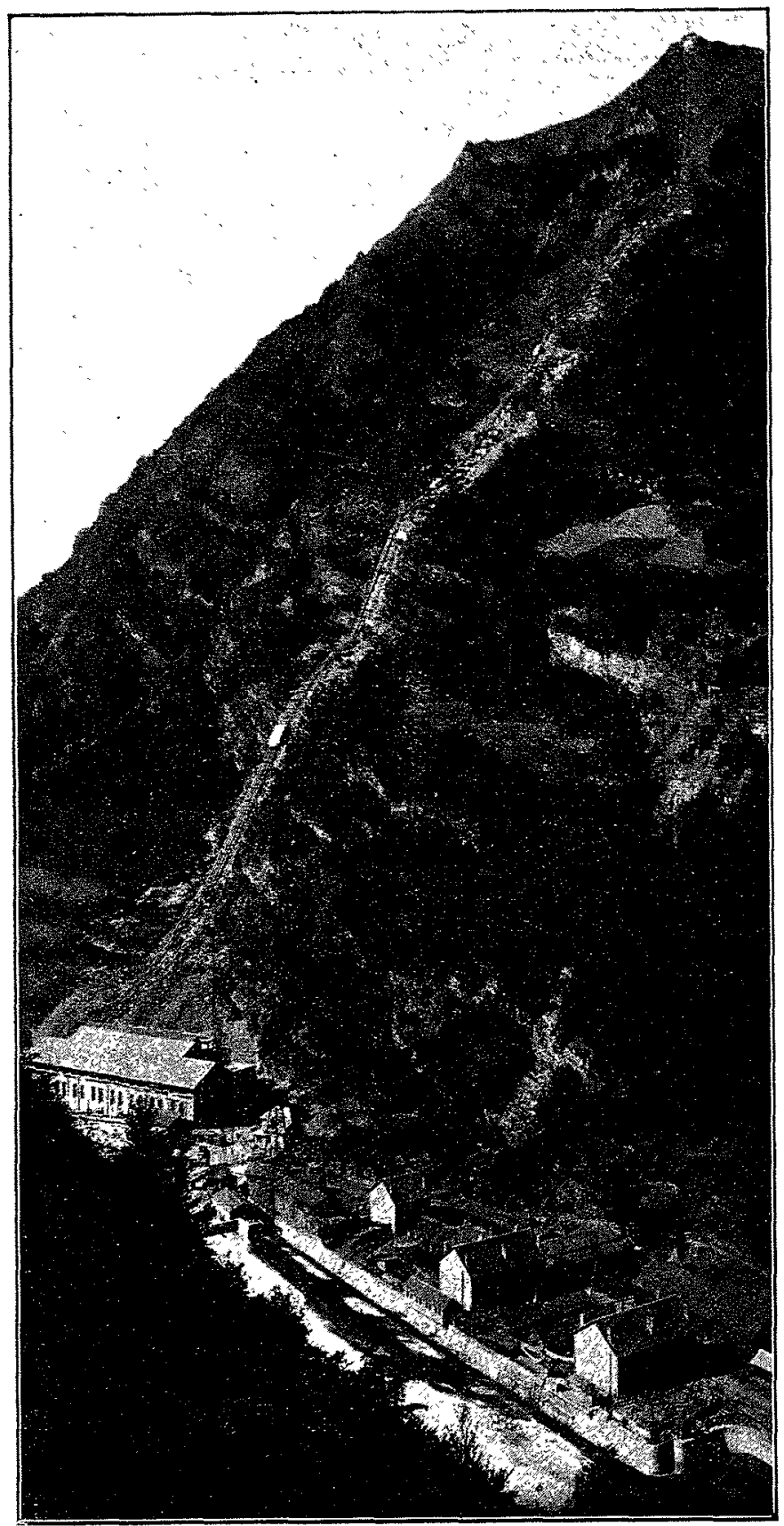

Compagnie des Chemins de fer du Midi. - Usine d'Eget

piliers généralement en maçonnerie espacés entre eux de 6 à 10 mètres suivant les longueurs des éléments de conduite, leur diamètre et leur épaisseur. Sauf dans le cas de conduite enterrée les tuyaux ne doivent pas reposer directement sur la maçonnerie. On interpose entre les luyaux el les piliers, des supports métalliques établis de telle façon que la conduite puisse glisser librement. En général, on utilise des supports en fonte ou sn tôle $\mathrm{e}^{\mathrm{n}}$ forme de berceau. Quelquefois, on prévoil de simples fers à $\iota$ scellés dans le pilier. Dans ee dernier cas, il laut vérifier que la conduite peut reposer sur une simple ligne sams risque de deformation.

Dans le cas de conduile enterrée el lorsque la chose peul se réaliser sans entraîner une dépense exagérée, il est bon de la faire reposer sur un lit de pierraille formant drain pour faciliter lécoulement des caux dinfiltration.

\section{Y. - DISPOSITIONS PARTICULALRLS}

Dans certains cas, on ne peut pas rapprocher suffisamment les supports intermédiaires parce que la conduite est obligée de franchir d'une seule portée, des creux ou des vallées.

Quelquefois on fait passer la conduite sur une passerelle métallique, sur un ponl en maçonnerie ou en ciment armé; d'autres fois, on la supporte par une simple ferme. Mais la solution la plus élégante qui, en outre, a l'avantage d'être très économique dans le cas où les porlées sont grandes el les tuyaux de gros diamètre, consiste à donner à la conduite la forme d'un arc qui se soutient de lui-mème. Nous connaissons plusieurs exemples de traversée de ce genre, deux surtout sont particulièrement impressionnantes :

Celle de l'usine de St-Jean-de-Maurienne où la conduite franchit "l'Arc " avec $3^{\mathrm{m}} 30$ de diamètre et 70 mètres de portée cl
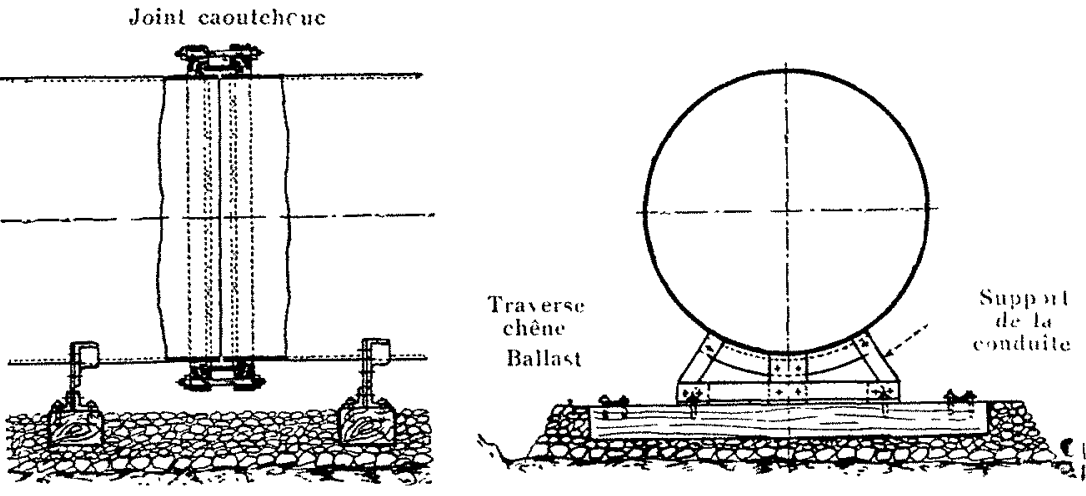

Fig. 4

celle de l'usine de l'Argentière-la-Bessée avec $2^{\mathrm{m}(6.5}$ de diamètre 65 mètres de portée en passant à près de 100 mètres au-dessus de la "Durance".

On arrive presque toujours à placer la conduite sur un sol stable. On peut cependant se trouver en présence de terrains mouvants nécessitant des travaux de fondation considérables pour obtenir une assise convenable. Nous avons eu à étudier, il y a peu de temps, ce cas particulier pour une conduite d'amenée destinée aux Usines Renault, à St-Michel-de-Maurienne.

Il a été solutionné de la façon suivante :

La conduite de $1 \mathrm{~m} 25$ de diamètre a été conslituée en luyaux soudée de 6 mètres de longueur réunis entre eux par un joinl élastique, dil "Universel ". (fig. 4), Elle repose sur le sol comme les voies ferrées par l'intermédiaire de supports métalliques, traverses de chênes el ballast. On a ainsi constilué un ouvrage dont l'ensemble est d'une flexibilité remarquable. Si le terrain vient à s'affaisser, la conduite se déforme sans aucun risque du fait de la nature des joints. Cette déformation est évidemment limitée el lorsqu'elle a alteint son maximum pratiquement possible; on procède au relevage de la conduite d'une façon absolument analogue au relevage des voies ferrées.

Lorsqu'on veut installer une conduite, on procède au relevé topographique exact du tracé, on détermine les terrassements 
it eflectuer et on exécute a l'atelier les luyaux avec leurs coudes de façon qu'au montage la conduite s'adapte parfaitement au profil de la platelorme préparée pour la recevoir. Il arrive parfois que le profil de la conduite est tellement accidenté qu'il devient presque impossible de faire un relevé suffisanment exacl et qu'on ne peut pas expédier de l'atelier les coudes préparés d'avance. M. Boucher, l'ingénieur suisse bien connu a solutionné la question (pour la chute d'Orlu de 940 mètres, dans l’Ariège) de la manière suivanle :

Tous les luyaux de la conduite sont des luyaux à brides : une des brides est normale à l'axe du luyau, l'autre bride est biaise. On conçoil qu'il est possible dans ces conditions, pour l'assemblage de deux brides biaises entre elles, soil de constituer des tronçons complétement droils soil d'obtenir des angles variant de $0^{\circ}$ au maximum dépendant du biais prévu sur les brides. On a pu ainsi oblenir des angles jusqu'à jo.torsque la nécessité s'est fait sentir on a également obtenu des angles analogues à la jonction des brides droites par l'interposition de deux faux joinls biais qu'on Gavait lourner lon par rapport à l'autre jusqu’à oblenir langle convenable.

De celle facon la conduile esl arrivée à épouser d'me laçon parfaite la plateforme préparée d'avance.

\section{Montakie Des connelTes}

Pour imstaller une conduile forcés suivant un tracé délerminé, on commence par débroussailler son emplacement el on procède au relevé très exact du profil en long du lerrain. Au bureau d'études ou élablit le tracé de la conduite sur le profil ainsi obtenu en ayant soin de réduire aulanl que possi-

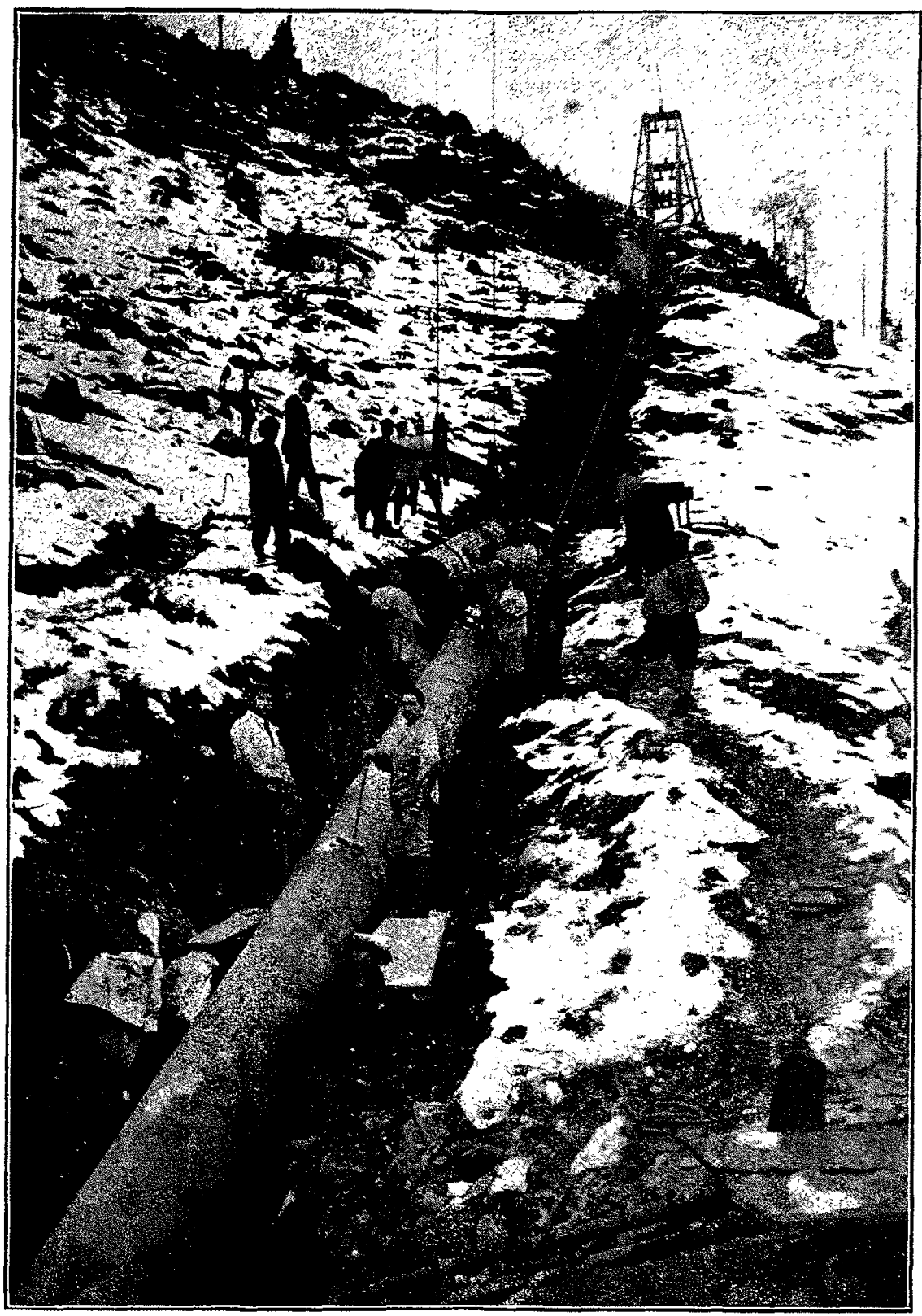

Montage par câble
Le montage proprement dit des conduiles est une opération difficile qui nécessile la mise en jeu d'un outillage et matériel importants el rui doit être exécuté par des spécialistes très expérimentés.

On conçoit aisément les difficultés qu'on peul rencontrer pour amener à leur point de montage et pour jonctionner entre eux des tuyaux pesant plusieurs tonnes le long des pentes arrivant parfois à se rapprocher de la verticale.

Les tuyaux sont d'abord camionnés jusqu'à la parie inférieure du tracé de la conduite. On procède ensuite à leur transport le long du profil ce qui s'effectue généralement de deux manières différentes

Lorsque le profil de la conduite n'est pas très accidenté (jusqu'à $50 \%$ de pente) on installe un plan incliné avec voie de $0^{\mathrm{m}} 60$ ou $1^{\mathrm{m}}$, selon les dimensions des tuyaux à transporter.

On les remorque (à l'aide d'un treuil mécanique et d'un câble tracteur guidé en plan el en élération par des dispositils appropriés) après les avoir fixés sur des wagonnets spéciaux articulés dans le sens horizontal et dans le sens vertical puis on procède à leur déchargement et bardage latéral ce qui n'est pas toujours une opération facile.

Cuand le profil est accidenlé, on installe un câble transporteur qui peut avoir de très grandes portées. Il est généralement constitué par un ou deux câbles porteurs dont les dimensions varient avec les charges à porter. Sur ces càbles porteurs roule un chariot spécial entraîné par un câble tracleur sans fin mû par un treuil mécanique. Le tuyau est suspendu à ce chariot par l'intermédiaire d'un câble moufleur également mû mécaniquentenl et constitué de telle façon qu'on puisse monter ou ble le nombre de troncons coudés sans exagérer, toutefois, l'imporLance des terrassements à effectuer. On trace le "fond de tranchée" qu'on lixe généralement à $0^{\mathrm{m}} 40$ environ au-dessous de la génératrice inférieure des tuyaux et on détermine les emplacements des piliers el des massifs d'ancrage. Sur le chantier on exécute en premier lieu les terrassements prévus sur les dessins; on corstruil ensuite les piliers el massifs jusqu'au niveau du fond de lranchée sculement. Ceux-bi ne sont terminés qu'après la mise en place definilive des luyaux: En cours de montzos exécute s'il y a lien des niches de dimensions envendes autour des joints pour faciliter la pose des rivets et le serrage des boulons. descendre la charge à volonté à n'importe quelle hauleur et à n'importe quel point du parcours.

Lönstallation d'un cable transporteur est beaucoup plus cô̂teuse que celle d'un plan incliné, mais elle a l'avantage de permettre une économie sensible de main-d'ourre dans la manutention des tuyaux el de supprimer les mancurres brutales qu'il est très difficile d'éviter aulrement.

Les tuyaux étant amenés à leur emplacement définitif par l'un des deux moyens ci-dessus, il ne reste plus qu à les emboiter, river et mater ou à procéder à la mise en place des joints et au serrage des houlons. Ces opérations quoique courantes nécessi- 
tent beaucoup d'expérience el d'habileté de la part des monteurs à cause des difficultés résultant des lieux de montage.

Lorsque le diamètre extérieur des conduites ne dépasse pas 2 m80 on expédie sans difficulté sur les chantiers des tronçons de conduite de 6 à 12 mètres de longueur selon les cas. De $2^{\text {m80 à }}$ $3^{\mathrm{m}} 30$, on peut encore opérer ainsi mais il faut utiliser des wagons agencés spécialement ce qui complique le problème des transports surtout si le lieu de pose est éloigné de l'atelier de fabrication.

Pour les conduites de plus de $3^{\text {n'}} 30$ de diamètre il n'est plus possible de constituer à l'atelier des tronçons de conduite. On expédie alors la fourniture en éléments démontés de dimensions convenables complètement préparés (percés, chanfreinés, cintrés et forgés). Ces éléments sont assemblés, rivés et matés sur place dans un véritable chantier de construction situé à proximité du lieu de montage. On constitue ainsi des tronçons comme on le fait dans le cas normal aux ateliers de construction et on procède à leur montage comme d'habitude. ralement pas bon usage, d'abord du fait des intempéries et ensuite parce que pendant la plus grande partie de l'année, clles se trouvent recouvertes d'une buée épaisse provenant de la condensation de la vapeur d'eau de l'air sur la paroi extérieure de la conduite.

\section{Mise en service et exploitation des conduites forcées}

\section{1. - NETTOYAGE PRÉLIMINAIRE}

Pendant le montage des tuyaux, il peut tomber à l'inlérieur de la conduite différents corps tels que : bois, rivets, boulons, outils, etc...

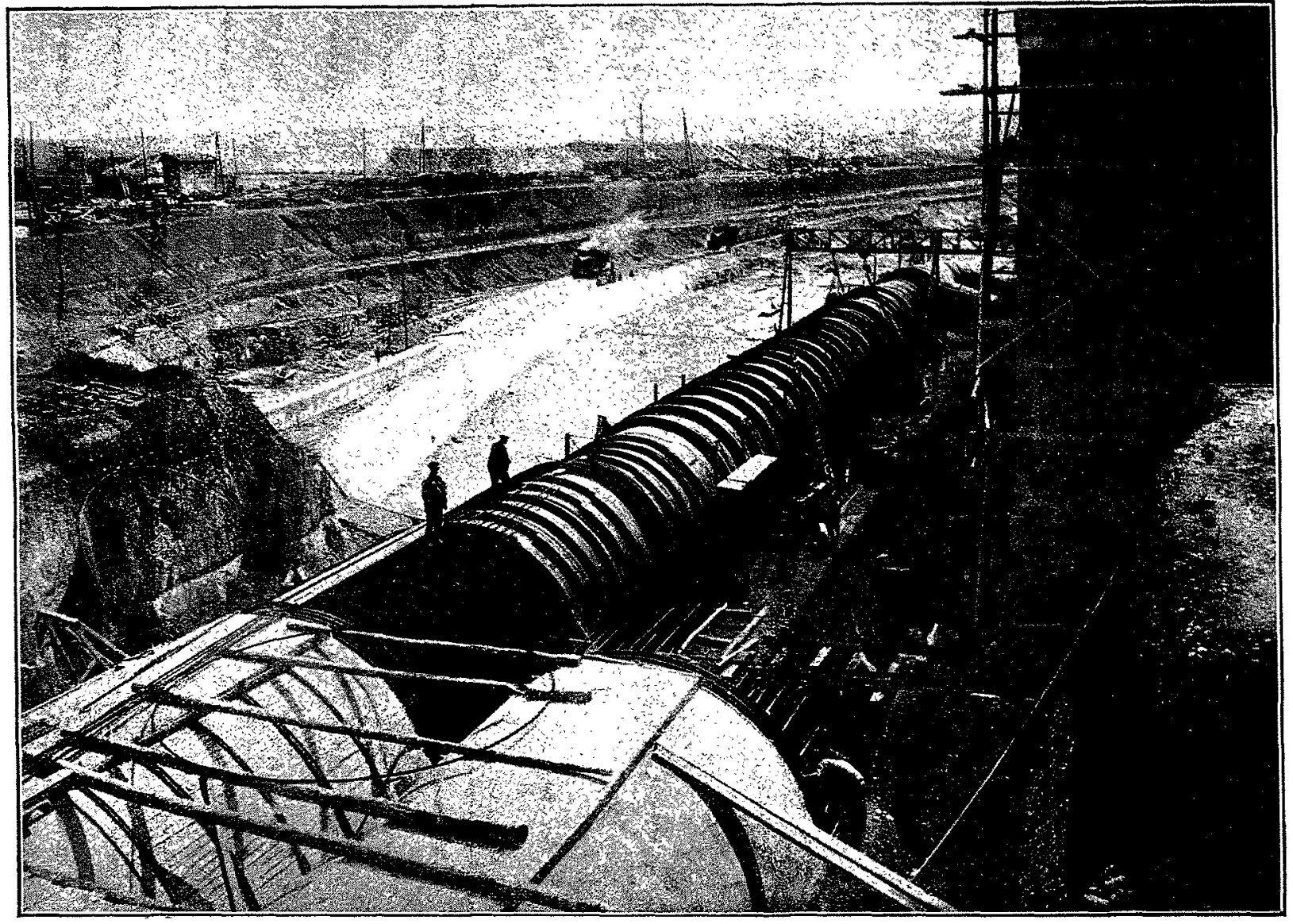

Energie électrique du Littoral Méditerranéen. - Collecteur de Sainte-Tulle

C'est ainsi qu'on a opéré pour les deux conduiles de SainteTulle (diamètre $4 \mathrm{~m} 50$ ) et pour les deux conduites d'Eguzon (diamètre $4^{\mathrm{m}} 25$ ).

\section{VII. - Peinture des CONduites}

Les tronçons de conduite sont généralement peints à l'atelier de construction à une couche de peinture au minium. Après leur mise en place, on les peint habituellement à deux couches de peinture au goudron pour les conduites enterrées et à une couche de peinture au minium et une couche de peinture claire pour les conduites à air libre. La peinture claire a pour but d'atténuer les effets des rayons solaires lorsque la conduite est vidée en été.

Dans le cas de conduite hors sol, on est obligé de reprendre les peintures assez fréquemment, les meilleures ne faisant géné-
Ces objets divers peuvent rester accrochés le long des fortes pentes ou rouler jusqu'aux parties en faible pente.

Il importe, avant la mise en service, de débarrasser complètement la conduite de ces corps étrangers qui pourraient, soit provoquer des coups de bélier en obturant brusquement les orifices d'admission des turbines, soit détériorer les turbines elles-mêmes.

Pour effectuer ce nettoyage, il faut d'abord ouvrir en grand le robinet de vidange situé généralement à l'aval du collecteur. Si ce collecteur est terminé par un fond à brides, il est préférable d'enlever ce fond et d'effectuer la chasse par l'orilice ainsi découvert.

On admet ensuite dans la conduite une certaine quantité 
d'eau qui coule en cascade et entraîne avec elle la plupart des corps étrangers.

Le lavage doit être très énergique et durer une heure environ et plus si cela est possible.

Ce nettoyage doit être complété par une visite intérieure de toutes les parties accessibles de la conduite. Il y a lieu d'examiner plus particulièrement : le collecteur, les parties en faible pente, les points bas s'il en existe et les tubulures.

\section{Les points bas se trouvent:}

$1^{\circ}$ Dans les siphons; $2^{\circ}$ Dans les traversées en arc; $3^{\circ}$ Dans les tubulures en dessous; $4^{\circ}$ Dans les raccords par dessous; $5^{\circ}$ Dans les robinets-vannes; $6^{\circ}$ Dans les obturateurs; $7^{\circ}$ Dans les collecteurs ; $8^{\circ}$ Dans les venturis.

\section{II. - REMPLISSAGE}

a) Lorsque le gel n'est pas à craindre.
La première mise en charge doit être effecluée très lentement. Le débit de remplissage doit être de l'ordre de $1 / 10$ du débit de fonctionnement.

Les mises en charges suivantes peuvent être effectuées plus rapidement.

Toutes les mises en charge, et principalement la première, doivent être surveillées très attentivement au manomètre branché sur le collecteur.

Un garde-vanne doit être à l'origine de la conduite pendant toute la durée de l'opération, prêt à couper le débit le cas échéant.

Lorsque, à l'amont de la conduite, il existe un robinet-vanne muni d'un by-pass, le remplissage doit être effectué au moyen de ce dernier.

On ferme les robinets d'évacuation d'air et les ventouses au moment où l'eau commence à s'échapper par leurs orifices.

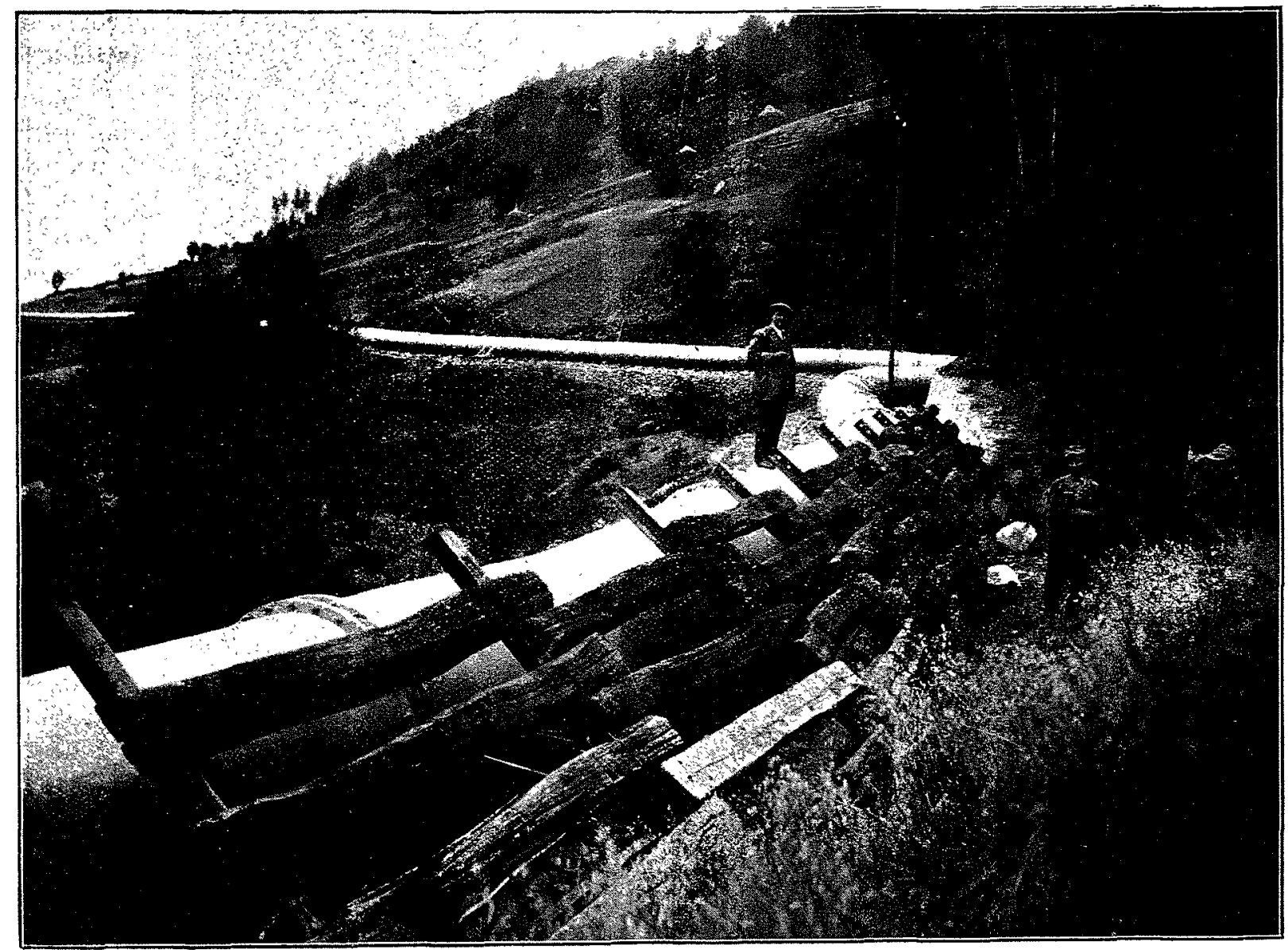

Usines Renault à Saint-Michel-de-Maurienne. - Conduite flexible

Il faul s'assurer, d'abord, que le manomètre enregistreur est en parfait état de fonctionnement.

On doit ensuite ouvrir tous les robinets d'évacuation d'air ainsi que les ventouses s'il en existe.

Les robinets d'évacuation d'air sont prévus sur tous les points hauts.

Ces points se trouvent :

$1^{\circ}$ Dans les siphons automaticques d'arrèt de débil; $2^{\circ}$ Dans les traversées en arc ; $3^{\circ}$ Dans les tubulures en dessus ; $4^{\circ}$ Dans les raccords par dessus; $5^{\circ}$ Dans les robinets et obturateurs; $6^{\circ}$ Dans les venturis; $7^{\circ}$ Dans les collecteurs; $8^{\circ}$ A l'aval des robinets-vannes; 90 A l'aval des vannes d'entrée.

On s'assure que les obturateurs sont en bonne position de fonctionnement.
La conduite est en état de fonctionnement lorsque le remplissage est terminé et que tout l'air contenu dans la conduite a été évacué.

\section{b) Lorsque le gel est à craindre.}

Dans ce cas, il faut, en premier lieu, s'assurer que toutes les parties de la conduite dans lesquelles le gel est possible sont suffisamment protégées. Ces parties dangereuses sont celles où l'eau est immobile ou en mouvement ralenti, telles que : tubulures, robinets-vannes, robinets de vidange, by-pass, culs-desac, etc...

On procède ensuite au remplissage en observant les règles énoncées pour le cas où le gel n'est pas à craindre, mais en ayant soin toutefois de laisser le robinet de vidange partiellement ouvert 
de façon à maintenir un éconlement. continu dans la conduite.

Le débit du robinet do vidange doit ètre maintenu au moins égal à 1 melre cube par heure el par mètre carré de surface de conduite mouillée ed exposée au relroidissement.

Dans ce cas le débil de remplissige admis à l'origine de la conduite doit ètre le mème que dans le premier cas augmenté du débit d'ecoulement du robinet de vidange.

\section{III.- VIDANGE}

La conduite peul ètre vidée soit au moyen des turbines, soit au moven du robinet de vidange du collecteur, soil par les deux ensemble.

Il faut, en premier lieu, fermer la ranne ou le robinet-vanne qui se trouve en tète de la conduite. Il faut ensuite faire fonctionner les ventouses à la main afin de s'assurer qu'aucun obstacle ne s'oppose à leur ouverture.

S'il y a un reniflard, il faut s'assurer qu'un bouchon de glace n'en obture pas l'orifice.

Les ventouses et les reniflards sont des organes qui permettent la rentrée automatique de l'air à l'intérieur de la conduite au moment d'une vidange de celle-ci.

Si un obstacle s'opposait à l'entrée de l'air à l'intérieur de la conduite, une dépression se produirait et la pression atmosphérique agissant à l'extérieur provoquerail, dans bien des cas, l'aplatissement des luyaux de mince épaisseur.

Cies précautions étant prises, on peut ouvrir le robinet de vidange et les turbines.

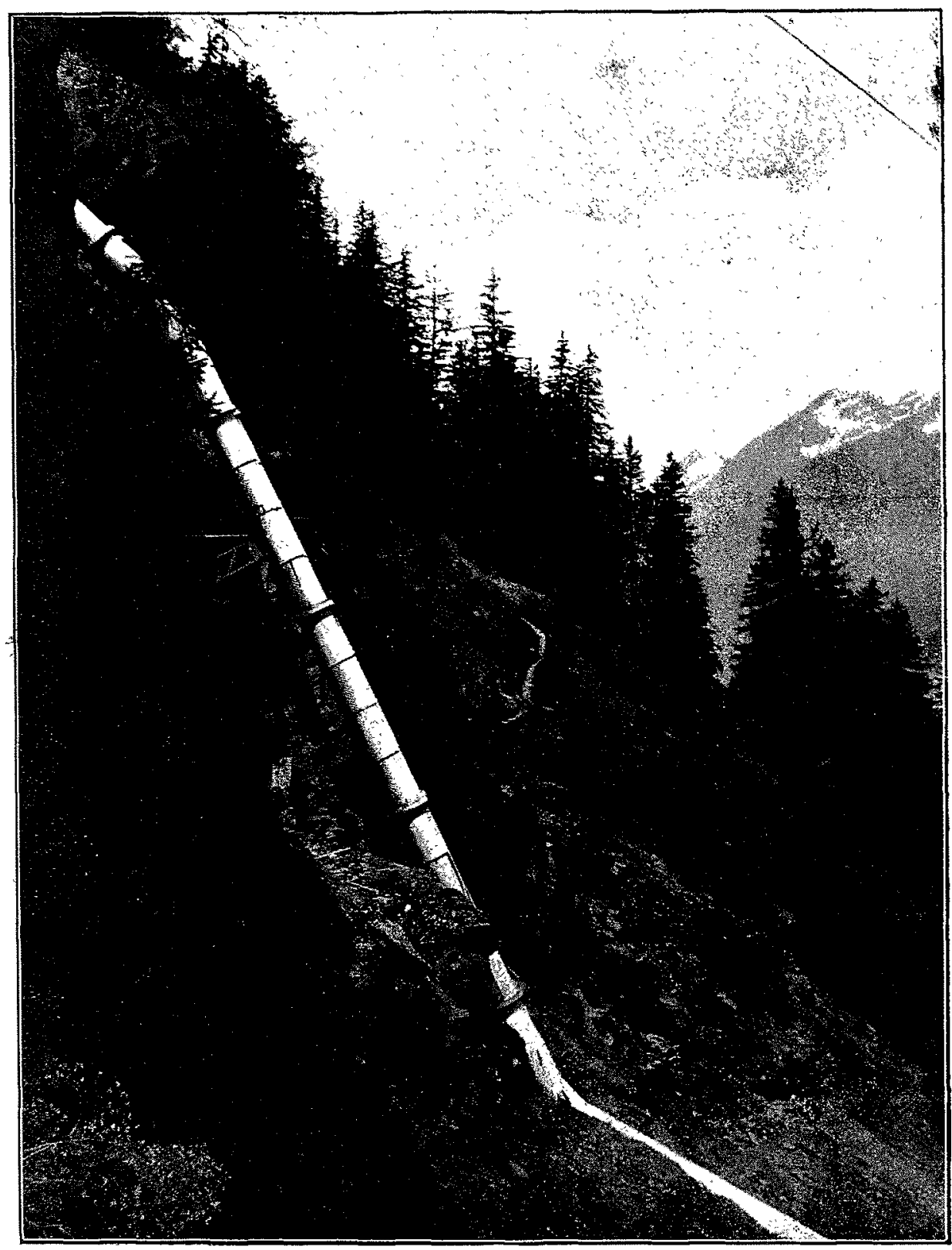

Jonction par brides dans une forte pente
Les joints à brides avec plomb et bitord peuvent être resserrés ou matés, si leur étanchéité laisse à désirer.

Les joints à emboîlement avec cordon de caoutchouc son $l$ généralement très étanches. Lorsque le cordon de caoulchouc a été mal placé, le joint perd en abondance et doit alors être refait.

Lorsque de petits suintements se produisent aux soudures des tuyaux soudés, cela résulle de soufflures sans importance.

Ces suintements peuvent aussi être observés sur les pièces moulées : tubulures, brides, coudes, etc., el proviennent également de soufflures.

Les petites fuites el suintements s'arrêtent d'eux-mèmes au bout d'un certain temps, car les matières en suspension dans l'eau produisent à la longue un colmatage naturel.

Il faut noter que tous les appareils de fermeture lels que : robinets-vannes, oblurateurs, vannes-papillon, etc., ne domnent qu'une étanchéilé relative. L'étanchéité parfaite et absolue n'est pour ainsi dire jamais obtenue avec ces appareils.

\section{V. -. PROTECTION}

CONTRE LE GIL.

Les conduiles forcées et leurs organes spéciaux doivent être protégés contre le gel.

Dans la tuyauterie proprem ent dite, l'eau peut geler si la vilesse est insuffisante. Cette vitesse minimum doil ètre calculée par celui qui dirige l'exploitation. Il laut dans ce calcul, tenir comple de l'altitude de la prise d'eau, de la longueur des souterrains et de
La vidange doit êlre suivie au manomètre sur lequel on peut juger de la rapidité de l'opération.

Il faut ensuite vidanger lous les points bas.

\section{IV. - ËTANChÉrté}

Lors de la première mise en charge, de nombreuses fuiles sont apparentes, soit aux lêtes de rivets, soit aux recouvrements des tôles. Ces fuites s'aveuglent au moy un d'un matage approprié.

Malgré un matage très soigné, il subsiste toujours de petils suintements qui s'arrêtent d'eux-mêmes par colmalage naturel. la surface de luyaux exposée au refroidissement.

Nous cilons la formule suivante qui semble applicable dans la plupart des cas :

Le débit minimum doit être au moins égal à 1 mètre cube par heure et par mètre carré de surface exposée au refroidissemenl.

Le gel ne paraît pas à craindre dans une conduite enterrée recouverte d'une couche de terre de $0^{\mathrm{n}} 80$.

'Toutes les parties de conduite dans lesquelles l'eau est en mouvement ralenti doivent être protégées. Ce sont : les corps de robinets, les tubulures dans lesquelles l'eau ne circule pas, les 
ventouses, oblurateurs, tuyauteries de manomètres, tuyauteries diverses, by-pass, robinets de vidange, chambres de prise de pression des Venturis. Il faul aussi protéger les collecleurs lorsque les turbines d'extrémilé sont arrêtées.

La protection peut être réalisée en plaçant autour des organes, à garantir, des coffrages garnis de fumier, paille, terre, ou de substances calorifuges.

Les parlies placées à l'intérieur d'un gros massif de maçonnerie paraissent à l'abri du gel.

Lorsque les appareils sonl situés à l'intérieur d'une chambre de mancuvre, il est recommandé de chauffer celle-ci par les grands froids.

Si la conduite proprement dite, ne peut être efficacement prolégée, il est indispensable de la vider complètement ainsi que les points bas, au cas où pour une raison quelconque, on se trouverait dans l'impossibilité de maintenir le débit nécessaire, soit par les lurbines, soil par le robinet de vidange.

\section{VI. - Dilatation}

Il faut, autant que possible, éviter de laisser les conduites vides au moment des grands froids ou des fortes chaleurs car c'est à ces moments que le travail est maximum dans le sens longitudinal de la conduite.

\section{VII. - Appareils} SPÉGIAUX

c) Vannes en tète des conduites. - Les vannes à tablier métallique (tôle et profilés) ne sont généralement pas étanches. On peut augmenter leur étanchéité en vidanl dans l'eau au-dessus du tablier, des scories pulvérisées, de la sciure de bois ou simplement de la terre.

Les vannes en fonte, à tablier el glissières rabotées, sont plus étanches, sans avoir pour cela une étanchéité parfaite. Leurs dimensions pratiques ne dépassent pas $1^{\mathrm{m}} \times 1^{\mathrm{m}}$. Elles ne peuvent donc être employées pour les grosses conduites.

d) Robinets-vannes et vannes papillons. - Ces appareils se construisent sans by-pass pour les petits diamètres et les faibles pressions. Si elle est très forte ou si le diamètre est grand, il est indispensable d'équilibrer la pression au moyen d'un by-pass.

Les robinets-vannes peuvent être employés pour les vidanges mais seulement pour les basses chutes.

Si la pression est élevée, il peut se produire des vibrations dangereuses de la lentille. Il est prudent dans ce cas de commencer la vidange par les turbines et de ne manceuvrer le robinet qu'au moment où la pression est devenue suffisamment faible.

Lorsque les robinets de vidange restent fermés très longtemps, il peut se former, contre la face amont de la lentille, un amoncellement de sable, qui, sous l'action de la pression peut constituer, à la longue, un bloc très dur. Afin d'éviter ceci, il est nécessaire de les manouvrer périodiquement de manière à produire un nettoyage constant. Les sièges en bronze de ces robinets sont très souvent rongés par le sable.

Les rannes papillons n'ayant aucun siège dressé, sont les appareils de fermeture les moins étanches. Il est généralement prévu, à l'aval de ceux-ci, un robinet d'évacuation des fuites.

e) Robinets à pointeau et robinets $\dot{a}$ cylindre équilibré. Ils sont habituellement employés pour les vidanges sous haute pression. Ils ont l'avantage de ne produire aucune vibralion. Leur mancuvre doit ètre périodique de façon à éviter l'accumulation du sable dans le corps des robinets.

Il faut éviter de les laisser en fonctionnement permanent. La vitesse à l'orifice de sortie atteint généralement une valeur considérable et l'usure des sièges est très rapide.

L'écoulement permanent ne doil ètre maintenu que dans des cas exceptionnels (période de gel par exemple).

f) Obturateurs. - Les obturateurs sont placés sur les collecteurs lorsque plusieurs conduites y aboutissent. Ils permettent d'isoler celles-ci.

En aucun cas ils ne doivent être manœuvrés si les conduites débitent.

Ils sont d'une étanchéité relative. Il est, par conséquent nécessaire de prévoir à l'amont un robinel de vidange destiné à l'évacuation des fuiles. 
Afin d'éviter toute manœuvre intempestive, il y a lieu d'amarrer solidement les leviers de manœuvre de ces appareils.

g) Venturis. - Les chambres de prise de pression communiquent avec la conduite elle-mème par l'intermédiaire de petits orifices circulaires.

Des incrustations peuvent boucher ces orifices et il est nécessaire de les nettoyer de temps à autre.

h) Ventouses. - Elles sont destinées à assurer automaliquement l'entrée de l'air dans la conduite au moment de la vidange de celle-ci. Elles peuvent aussi être mano'uvrées à la main.

Une cerlaine quantité d'eau s'échappe au moment de leur fonctionnement et il est nécessaire d'en assurer l'évacuation.

Les ventouses sont aussi utilisées pour l'évacuation de l'air au moment du remplissage.

Ces appareils doivent être visités périodiquement et on doit s'assurer par une manœuvre à la main de leur bon fonctionnement.

Lorsque les ventouses sont placées dans une chambre de manœuvre, il est nécessaire de prévoir, à travers le mur de celle-ci, un orifice toujours ouvert et d'une section suffisante pour le passage de l'air. Cet orifice sera toujours prévu de façon qu'il ne risque jamais de s'obstruer.

Il faut bien observer que le non fonctionnement des ventouses peut provoquer, au moment de la vidange, l'aplatissement de la partie amont de la conduite.

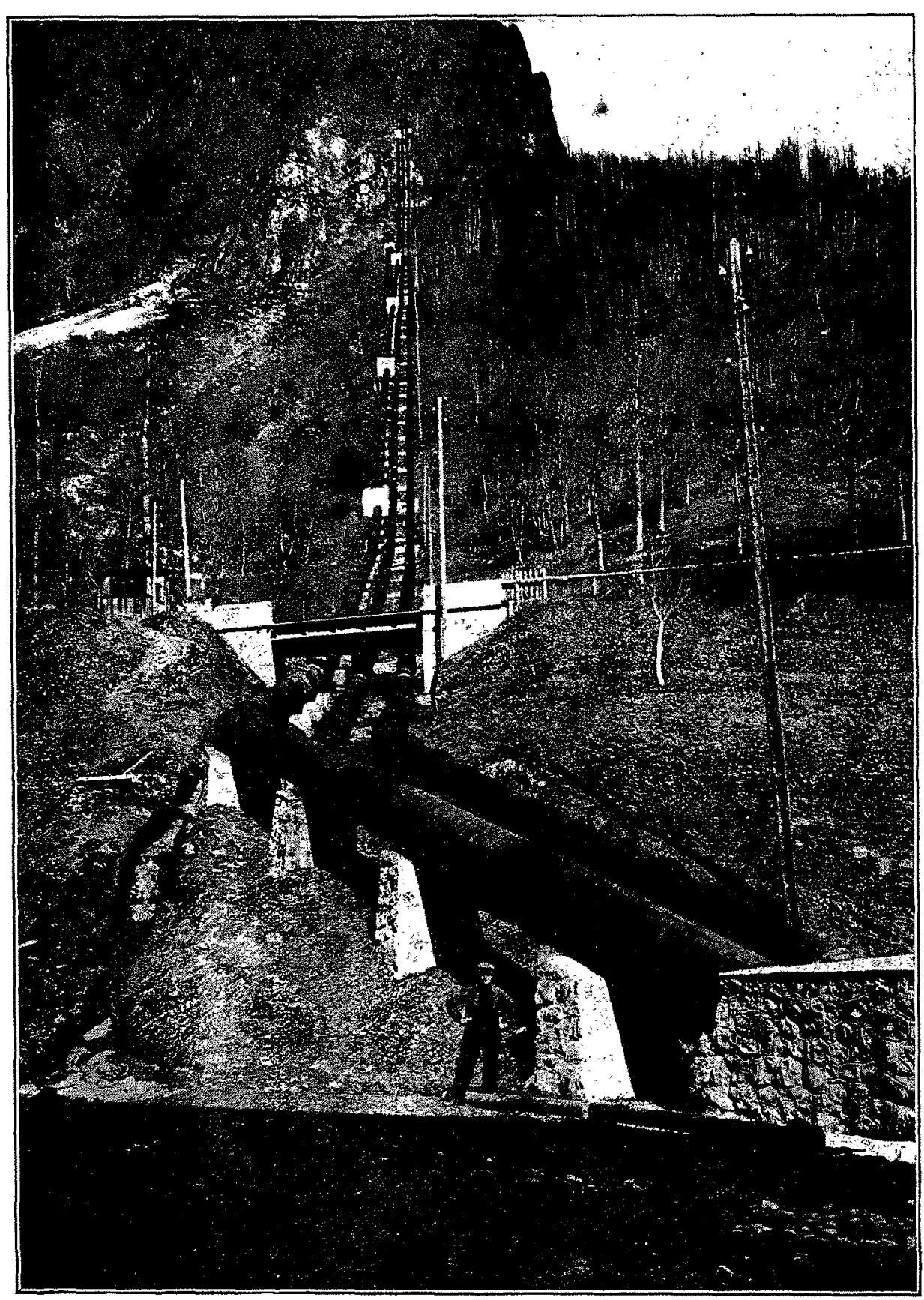

Société Hydro-Electrique de l'lau-d'Olle. Conduites forcées du V'ernay
Ils jouent le même rôle que les ventouses et doivent par conséquent ètre protégés sérieusement contre le gel.

j) Manomitres enregistreurs. - Ils sont placés à l'intérieur de la centrale et sont reliés au collecteur par une tuyauterie de petit diamètre qui doit être protégée contre le gel.

La prise sur le collecteur doit être faite en un poinl ne présentant pas de perte de charge singulière et sur le côté ou sur le haut du tuyau de façon à ne pas être obturée par le sable.

Sur la tuyauterie doit être branché un robinet à bride porteétalon, destiné à la vérificalion et à l'élalonnage.

k) Siphons aulomaliques. - Ces appareils sont du type breveté des Elablissement: Bonchayer \& Viallol.

Ils onl pour but de couper nel le débil des conduites lorsque la vitesse de l'eau dépasse une cerlaine limile. Cetle vitesse, pour laquelle l'appareil est calcule, est habituellement les $15 / 10$ de la vitesse normale.

L'amorçage peut être obtenu soit par surélévation momentanée du plan d'eau, soit au moyen d'un éjecteur produisant le vide.

$\mathrm{Si}$ une rupture se produit le long de la conduite, le débil augmente rapidement el, lorsque la limite admise est atteinte le siphon se désamorce, coupant ainsi automaliquement le débil.

I se débil de fonclionnoment ne peul pas être garanti très exactement car il est déterminé au moyen d'un calcul de pertes de charges par l'emploi des formules usitées.
Elles doivent être soigneusement prolégées contre le gel.

i) Reniflards. - Les reniflards doivent être prévus suffisamment élevés par rapport au niveau statique pour qu'à la fermeture des turbines il ne se produise pas de projections d'eau par leur orifice.

set orifice est généralement garanli au moyen d'une loile métallique, contre les pierres, bois ou autres corps que pourraient jeter les gens mal intentionnés.
Pour éviler le désamorçage intempestif de cet appareil en marche normale, il est indispensable d'évacuer périodiquement l'air entraîné par l'eau, qui arrive à s'accumuler au sommet du siphon. Il suffit de faire fonctionner tous les jours pendant quelques minutes, l'éjecteur d'amorçage

1) Vunnes papillons aulomaliques d'arrếl de débil. - Ces appareils remplissent le même bul que les siphons automatiques d'arrêt de débil. 
Ils sont d'un fonctionnement moins sûr que ces derniers, étant donné les organes mécaniques qui entrent dans leur construction.

Ces papillons sont généralement précédés d'une vanne ou d'un robinet-vanne. Leurs organes sont d'un fonctionnement assez délicat aussi doit-on les visiter, les faire fonctionner périodiquement et les entretenir soigneusement graissés.

m) Détendeurs de pression. - Les détendeurs sont installés le plus souvent à l'intérieur des centrales. Ils sont placés sur les tuyauteries de refroidissement des transformateurs et des paliers, les tuyauteries d'incendie, les tuyauteries d'alimentation de parafoudres.

Ils doivent, par conséquent, assurer un débit constant.

Mais ils sont très délicats et les feuilles, bois, etc., qui sont en suspension dans l'eau arrivent à les obstruer assez rapidement.

Un démontage s'impose pour le nettoyage du détendeur et à cet effet, un by-pass est généralement prévu pour assurer le fonctionnement pendant cette opération. Si l'eau contient trop d'impuretés il est indispensable de prévoir un filtre muni luimême d'un by-pass de nettoyage.

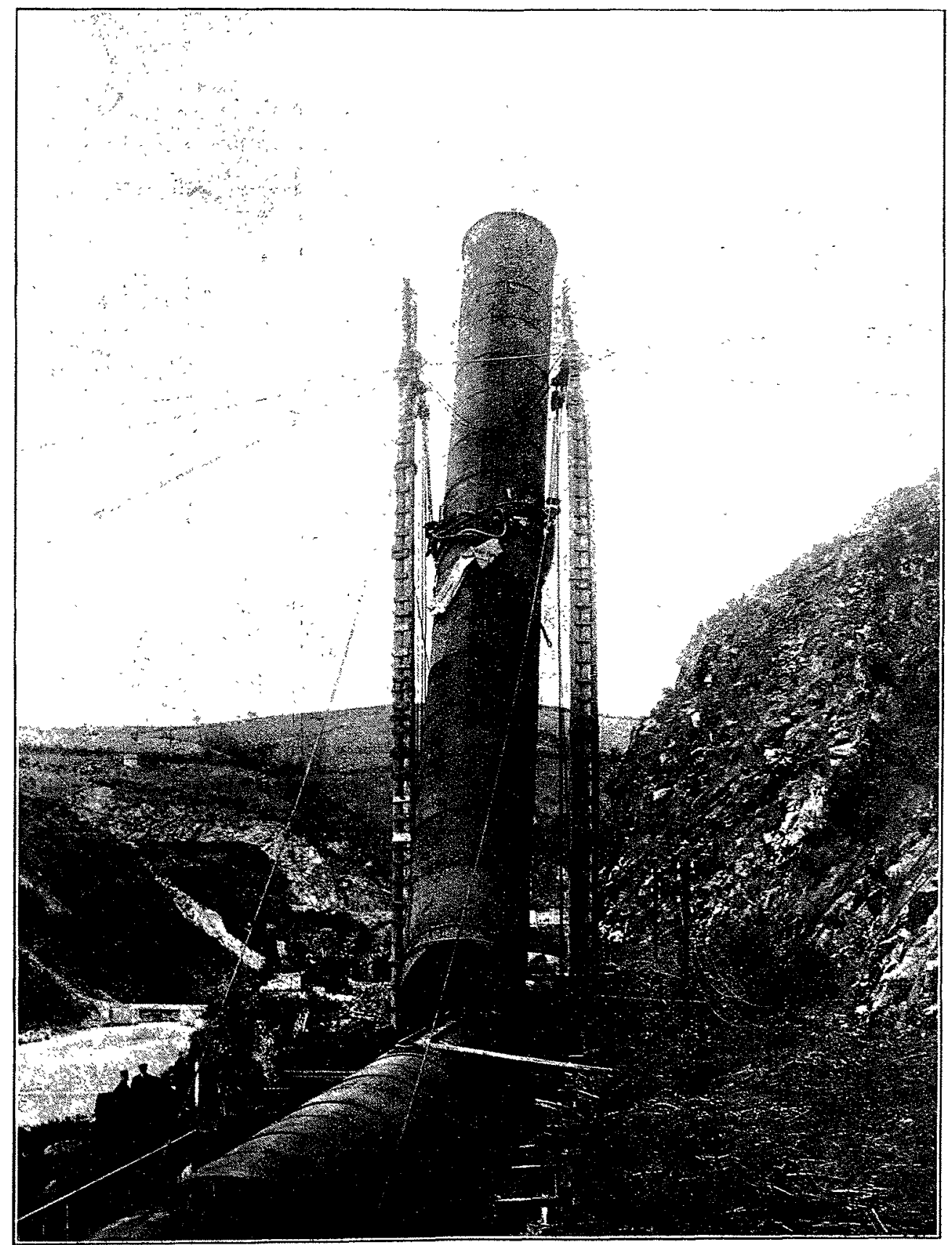

Nontage d'une cheminée d'équilibre 